\title{
Variables morfológicas en el procesamiento del lenguaje
}

\author{
Susana del Viso* \\ José E. García-Albea
}

Universidad Complutense

\section{INTRODUCCION}

La capacidad de utilización del idioma - a efectos de comprensión y producción- por parte de los hablantes de una lengua implica, por un lado, la existencia de un almacén de información altamente estructurado y con unas determinadas propiedades de organización $\mathrm{y}$, por otro, la de un sistema de recuperación que parece disponer de un procedimiento de búsqueda extremadamente rápido y eficaz. Saber cómo está organizado el sistema de almacenamiento y cómo operan los procesos de recuperación reviste una importancia capital para una teoría del procesamiento del lenguaje, para una teoría de la comprensión y producción de oraciones, dado que comprender su significado exige recuperar información acerca de cada una de las palabras que las componen; y en cuanto a la producción, es evidente que no puede tener lugar hasta haber localizado las palabras que van a integrar las oraciones por las que se expresa el mensaje.

Todo este enfoque parte, indirectamente, del trabajo de R. C. Oldfield (1966), que fue quien desarrolló la noción de «diccionario mental" y se cuestionó cómo se recupera la información acerca de una palabra.

Este diccionario mental, también denominado léxico interno,

* Dirección: Departamento de Psicologia Experimental (D-3034). Facultad de Psicologia. Universidad Complutense. Madrid-1 I. 
consiste en una memoria específica de palabras que contiene información fonológica, morfológica, sintáctica y semántica relativa a las mismas. Las unidades que lo componen reciben el nombre de entradas léxicas, que serian, pues, representaciones mentales de las palabras.

El creciente interés por el estudio del componente léxico en el procesamiento del lenguaje se apoya en varios factores que ya se han revisado en otro lugar (García-Albea, 1982). Dentro de esta corriente, las distintas investigaciones tienen la finalidad de responder a una o varias de las siguientes cuestiones: a) ¿cómo está organizado ese supuesto léxico interno?, b) ¿cómo se representa la información contenida en el mismo? y c) ¿cómo se accede al léxico, es decir, cómo se recupera la información en él contenida? Más concretamente, se ha comenzado a estudiar el papel que cumplen distintas variables estructurales (fonológicas, ortográficas, morfológicas y sintácticas) en el procesamiento de palabras, de cara tanto a la forma de organización y representación de las mismas como a las estrategias de análisis del «input) verbal (por ejemplo, García-Albea, I 980, 1984; García-Albea, Sánchez-Casas y Del Viso, 1982; Sánchez-Casas, 1982; Taft, 1979 a, I 979 b; Taft y Forster, 1975, 1976).

Parece que para reconocer una palabra (situándonos en el caso de la comprensión) debe emparejarse el «input» sensorial con algún tipo de representación interna. Uno de los aspectos claves en el estudio de la organización del léxico interno se refiere a la forma en que se representa la información y a las posibles recodificaciones que podrían llevarse a cabo para que el acceso tenga lugar. Por ejemplo, ha habido numerosas discusiones acerca del si el reconocimiento de una palabra se basa en los rasgos globales de la misma o en rasgos individuales de sus componentes.(por ejemplo, Wheeler, 1970). Parece probado que el reconocimiento tiene lugar a través de unidades menores que la palabra y lo que se trata de determinar es cuáles podrían ser. éstas. Se han propuesto distintas opciones; por ejemplo, se ha postulado que tales unidades podrían consistir en los fonemas o letras - según se trate de reconocimiento auditivo o visual - (Massaro, 1974; Massaro, et al., 1980), la sílaba (Mehler, et al., 1981; Spoehr y Smith, 1973) y el morfema (Jarvella y Snodgrass, 1974; Rubin, Becker y Freeman, 1979; Smith y Sterling, I982; Taft y Forster, 1975, 1976).

Es nuestro propósito revisar aqui las investigaciones sobre el papel que pueden desempeñar variables de tipo morfológico en la organización y en el acceso al léxico interno.

\section{EL PAPEL DE LA ESTRUCTURA MORFOLOGICA EN EL PROCESAMIENTO DEL LENGUAJE}

Desde el punto de vista lingüístico, es evidente que, por ejemplo, la palabra enjabonado está compuesta por el morfema base jabón, el prefijo en y el sufijo adjetival ado. Desde una perspectiva psicológica, esto podria darnos pistas sobre la organización del léxico interno. Es posible, en este sentido, que el léxico esté 
organizado de tal modo.que enjabonado se almacene junto con jabón, jabonera, etc. Esto supone que no existiría una entrada léxica independiente para las palabras enjabonado o jabonera, sino que se almacenarian en la entrada «jabón», correspondiente a la forma base. A partir de aquí, Taft y Forster (i975) - cuyos trabajos comentaremos más detenidamente en el siguiente apartado- propusieron que el reconocimiento de una palabra (por ejemplo, enjabonado) exige un análisis morfológico previo; es decir, requiere eliminar el prefijo en y el sufijo -ado antes de que se pueda acceder a la representación léxica de la palabra en cuestión a través de la entrada «jabón». De modo similar, la palabra gatos estaría almacenada como "gato", siendo reconocida como palabra solamente después de haber eliminado la -s como morfema de plural. (Obsérvese que en el caso de enjabonado se trata de una palabra derivada, compuesta por prefijo + forma base + sufijo derivativo, mientras que gatos es una palabra no derivada, sino solamente con una inflexión: forma base + sufijo inflexivo.)

Hablando concretamente de la relación derivativa (aunque también puede hacerse extensible a la inflexión), Bradley (1979) propone que la consecuencia de la hipótesis que acabamos de exponer sobre el formato de la representación léxica sería la simplificación del inventario mental. Esto parece bastante plausible si pensamos que una misma entrada léxica serviría como representación tanto de las formas base como de las formas derivadas (o inflexivas). Esta es una idea, no obstante, que no ha partido de Bradley, sino que ella recoge de la literatura anterior (por ejemplo, Mac Kay, 1976, 1978; Manelis y Tharp, 1977) para desarrollaria con mayor profundidad. En este sentido, fundamenta la noción de simplificación del léxico apoyándose en la siguiente idea: la localización de una forma derivada en la misma entrada léxica que también sirve para la forma base correspondiente explica fácilmente la regularidad fonológica, sintáctica y semántica que se observa entre las palabras relacionadas morfológicamente e indica también la relevancia computacional de esta regularidad.

En cualquier caso, esta hipótesis no sólo se sustenta en argumentaciones lógicas, sino que ha recibido un considerable apoyo experimental en las distintas áreas psicolingüísticas. Los datos procedentes de los estudios sobre comprensión, producción, adquisición y patología del lenguaje vienen a complementarse unos a otros, pero, por razones de organización, los expondremos por separado en cuatro apartados, correspondientes a cada una de estas áreas. Nos extenderemos más en el área de la comprensión, por haberse centrado en ella, hasta el momento, nuestros trabajos.

\section{DATOS EXPERIMENTALES EN EL AREA DE LA COMPRENSION}

En el campo de la comprensión, las primeras investigaciones sobre el papel de los morfemas en el procesamiento de palabras (Gibson y Guinet, 1971; Kintsch, 1972; Snodgrass y Jarvella, 1972; Murrell y Morton, I974) son consistentes con la idea de que las 
palabras con afijos - o, al menos, las palabras con sufijos- están almacenadas en el léxico según su forma base.

Gibson y Guinet (197I) compararon el reconocimiento taquistoscópico de palabras con sufijos y sin sufijos. Los autores esperaban que se diera un incremento en el número de letras informadas en el caso de las palabras con sufijos en relación con las palabras sin sufijos, basándose en el supuesto de que los sufijos se procesan como unidades independientes. Pero no encontraron tal diferencia. Concluyeron que las palabras se leen como complejos de rasgos que se procesan independientemente. Sus resultados son consistentes con la idea de que las palabras con sufijos se almacenan en el léxico según su forma base. Snodgrass y Jarvella (1972), utilizando una tarea de decisión léxica, también observaron un incremento en los tiempos de reacción correspondientes a las palabras con afijos.

Kintsch (1972) realizó varios experimentos para comparar la dificultad de las palabras base y derivadas en una serie de paradigmas experimentales. Encontró que los nombres derivados de verbos se recordaban peor que los nombres base en una tarea de pares asociados.

Murrell y Morton (1974) encontraron diferencias en el reconocimiento taquistoscópico que estaban en función de la estructura morfológica. En este estudio se presentaba a los sujetos una lista de palabras a memorizar y, a continuación, se les hacía una prueba con palabras que: 1 ) eran idénticas a algunas de las palabras de la lista que habían memorizado, 2) compartian el mismo morfema base con algunas de las palabras memorizadas, 3) tenían las mismas letras iniciales que algunas de estas palabras y 4) no tenian relación con ninguna de las palabras de la lista. En general, el reconocimiento taquistoscópico mejor fue para la condición de palabra idéntica (condición 1); el reconocimiento intermedio correspondió a la condición 2; el peor se dio en las condiciones 3 y 4 . El hecho de que el reconocimiento en la condición 2 fuese mejor que en la 3 se interpretó en apoyo de un proceso de reconocimiento de palabras basado en el morfema. Al observar que el reconocimiento de la palabra car se veía facilitado por la memorización previa de cars y no por la de card, los autores llegaron a la conclusión de que la variable responsable de esta diferencia era la estructura morfológica y no la mera semejanza ortográfica o fonológica.

Tomando en consideración todos estos resultados, Taft y Forster (1975, 1976) los integraron en la formulación de un modelo del procesamiento de palabras basado en el morfema como unidad de análisis. Estos autores fueron quienes propusieron de un modo preciso y sistemático la hipótesis que antes hemos expuesto. Como se recordará, básicamente consiste en que el estímulo se analiza en sus componentes morfológicos antes de que tenga lugar la búsqueda en la memoria. Durante la búsqueda, la raíz de la palabra sirve como unidad de acceso. Por ejemplo, la palabra con prefijo recast se analizará como re- y -cast, llevando a cabo a continuación una búsqueda de la representación de la raíz en la memoria léxica. Una vez se encuentra ésta, se comprueba si la combinación prefijo-raiz es apropiada; así se distinguen los prefijos válidos de los que no lo son 
(por ejemplo, incast). También puede haber procesos adicionales que coordinen el significado del prefijo y de la raíz.

A partir de estos resultados, obtenidos a través de tres experimentos de cuidado diseño, Taft y Forster (1975) elaboran un modelo de reconocimiento de palabras que incorpora el proceso de descomposición morfológica. En la figura i se presenta un esquema de este modelo.

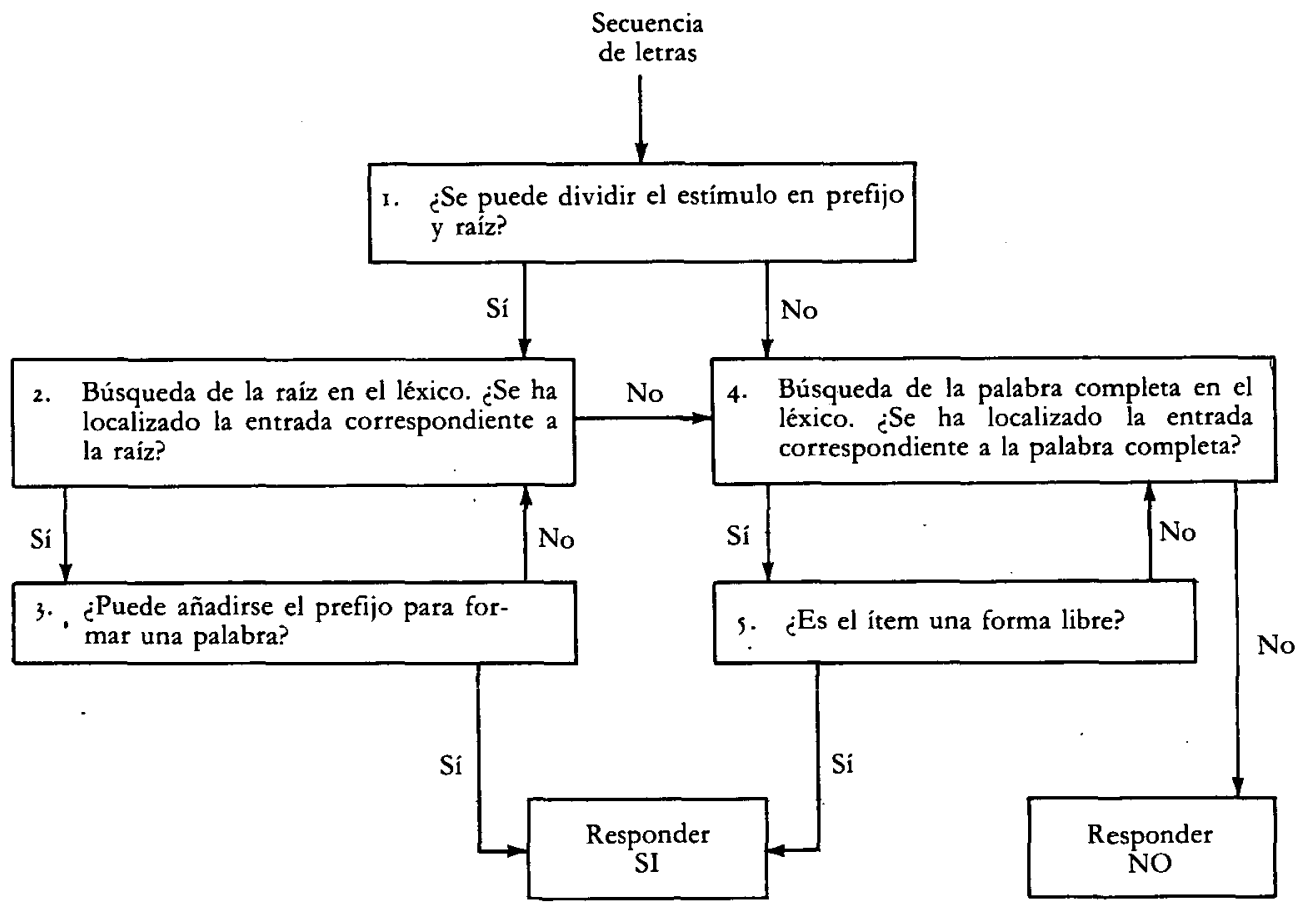

FigurA I

Modelo de reconocimiento de palabras basado en la descomposición morfológica, de Taft y Forster (1975)

Posteriormente, Taft y Forster (1976) realizaron cinco experimentos para determinar cómo están representadas las palabras polimorfémicas y polisílabas y cómo se accede a ellas. Partiendo de que a una palabra polimorfémica se accede a través de su raíz, los autores pretenden averiguar qué sucedería en el caso de que esa palabra estuviese compuesta por dos raices, como ocurre en las palabras compuestas. Las conclusiones que obtuvieron pueden resumirse en las siguientes: I) La entrada léxica de una palabra polisílaba se accede a través de una representación de su primera sílaba, independientemente de si ésta constituye una palabra o no; la información contenida en la entrada léxica especifica las posibles combinaciones de letras que pueden seguir a la primera sílaba. 2) El almacenamiento de las palabras polisílabas es el mismo, independientemente de cuántos morfemas posea la palabra; no obstante, en primer lugar, han de aislarse los posibles prefijos, es decir, una vez 
que se determina que la primera sílaba es un prefijo, comienza de nuevo la búsqueda a partir de la primera letra después del prefijo. 3) Para determinar la primera sílaba de una palabra, se inicia la búsqueda de todas las combinaciones de letras de izquierda a derecha hasta encontrar la entrada léxica apropiada.

En un trabajo más reciente, Taft ( 1979 b) estudió el reconocimiento de palabras con afijos utilizando el efecto de la frecuencia de uso como variable diagnóstica para determinar si este tipo de palabras poseen una representación léxica común con su forma base. En el primer experimento, estudió palabras con prefijos; en los dos restantes, palabras con inflexiones. En los dos primeros experimentos, comparó dos tipos de palabras, emparejando éstas - dentro de cada condición- en frecuencia particular y variando la frecuencia de las raíces respectivas o frecuencia total. Los resultados indicaron que los tiempos de reacción estaban en función de la frecuencia total, lo cual indica que las palabras relacionadas morfológicamente se almacenan juntas en el léxico, siendo este dato consistente con los de las investigaciones anteriores.

Sin embargo, en el tercer experimento se-demostró-que cuando--. la frecuencia total se mantiene constante y lo que se varía es la particular, los tiempos de decisión léxica se ven influidos por la frecuencia particular. Para explicar este resultado, Taft propone un modelo donde la frecuencia de uso ejerce sus efectos en dos fases distintas del proceso de reconocimiento. Concretamente, Taft recurre al modelo de acceso al léxico de Forster (1976). Lo que Taft postula es que las palabras están almacenadas en su forma base en los archivos de acceso periféricos y en su forma particular (final) en el archivo principal o léxico interno. (Para una discusión más detallada, véase Taft, $1979 \mathrm{~b}$, págs. 269-271).

Otros autores han obtenido resultados que van en esta misma línea. Por ejemplo, Lukatela, et al (1978), investigaron el «status» léxico de las palabras con inflexiones en el servocroata. Utilizando una tarea de decisión léxica, observaron que los nombres presentados en nominativo singular eran reconocidos en menos tiempo que el resto de los casos gramaticales (genitivo, acusativo, etc.). La conclusión que obtuvieron es que los nominativos singulares están ordenados en el léxico según la frecuencia, mientras que los otros casos gramaticales constituyen subentradas del nominativo singular de los nombres.

Aunque existen muchas investigaciones a favor de la hipótesis de la descomposición morfológica, no quisiéramos dejar de mencionar el hecho de que también se ha defendido la hipótesis contraria (por ejemplo, Holyak, Glass y Mah, 1976). Según ésta, las palabras con afijos poseerían entradas léxicas independientes de las correspondientes a sus formas base; el acceso a dichas entradas sería suficiente para reconocer las palabras. Los defensores de esta hipótesis esgrimen el argumento de que, dado que cada palabra sería reconocida como una unidad, esto supondría una economía en el procesamiento. Si bien, también podriamos argumentar que, por la misma razón, se requeriría un mayor número de entradas, lo cual implicaria un coste de almacenamiento adicional. 
En apoyo de esta hipótesis, Manelis y Tharp (1977) piensan que es posible que tenga lugar un análisis morfológico en el reconocimiento de palabras con prefijos, pero presentan dos experimentos en los que concluyen que tal análisis no tiene lugar en el caso de las palabras con sufijos.

En el primer experimento, Manelis y Tharp (1977) emplearon la tarea de doble decisión léxica, en la cual se presentan dos estímulos en cada ensayo y el sujeto tiene que decidir si ambos son plabras o si, por el contrario, uno de ellos es una no-palabra (o pseudopalabra). Utilizaron dos tipos de palabras: con sufijos (por ejemplo, tester, milky) y palabras sin sufijos, pero con la misma estructura (por ejemplo, sister, candy). Si se lleva a cabo una descomposición morfológica en el reconocimiento, sister sería erróneamente analizada como "more sist» o "someone who sists»; por esta razón, las palabras sin sufijos tardarían más en reconocerse que las que tienen sufijos. Sin embargo, los autores no encontraron diferencias en los tiempos de reacción (TR) ante dos palabras sin sufijo (por ejemplo, sister somber) comparados con los TR empleados en responder a dos palabras con sufijo (por ejemplo, tester sender), si bien estas dos condiciones dieron lugar a tiempos de respuesta menores que cuando se combinaban en una misma presentación una palabra con sufijo y otra sin él (por ejemplo, tester somber).

De todos modos, estos resultados distan mucho de ser concluyentes en favor de una hipótesis basada en la no descomposición morfológica. Como explica Taft (1979 b, págs. 266-267), el resultado de que esta última condición resultase más difícil que cualquiera de las dos anteriores (donde las dos palabras presentadas simultáneamente eran del mismo tipo) lo que, de hecho, apoya es la hipótesis de la descomposición: cuando un mismo grupo de letras (por ejemplo, -er) cumple una función morfológica distinta en cada miembro de un par de estímulos, el conflicto que se produce podría elevar el tiempo de decisión, pero solamente si las palabras en cuestión se analizan morfológicamente. Además, parece que habia algunos sesgos en la selección de los estímulos (véase Taft, 1979 b, pág. 267).

En el mismo artículo, Manelis y Tharp llevan a cabo un segundo experimento. En este caso, se presentaba a los sujetos una palabra (por ejemplo, snow) que pudiera servir como forma base en una palabra con sufijo. A continuación, se presentaba una palabra o una no-palabra con sufijo. La tarea del sujeto consistía en decidir si la palabra presentada en primer lugar estaba contenida o no en el item que seguía (por ejemplo, "SI" para snowed y snowen; "NO» para slowed y slowen). Los resultados indicaron que se tardaba más tiempo en reconocer la palabra base en una no-palabra que en una palabra. Los autores argumentan que, si hubiera tenido lugar una descomposición morfológica, los sujetos habrían encontrado snow en snowen aproximadamente en el mismo tiempo que en snowed, dado que la descomposición ha de llevarse a cabo previamente al acceso léxico.

Pero también en este caso existe un serio problema en relación con los materiales empleados en el experimento: la distribución de los sufijos que se añadieron para formar palabras fue en gran medida 
diferente de la distribución de los que se añadieron para componer las no-palabras. Los sufijos más comunes en inglés (como -ing, -er y -ed) aparecían en 80 de las 96 palabras y en ninguna no-palabra. En cambio, sufijos mucho menos frecuentes (como -est y -en) aparecian en 62 de las 96 no-palabras y en ninguna palabra. Este desequilibrio en la frecuencia de los sufijos utilizados podría explicar los mayores TRs ante las no-palabras, ya que no es descabellado pensar que el reconocimiento de los sufijos pudiera verse influido por la frecuencia de éstos.

En cualquier caso, el experimento 2 de Taft (1979 b) parece demostrar que los sufijos inflexivos (al igual que los prefijos) son eliminados antes de iniciar la búsqueda léxica de la representación de la raíz.

Algo más recientemente (Stanners, Neiser y Painton, 1979; Rubin, Becker y Freeman, I979), el modelo de Taft y Forster se ha seguido poniendo en tela de juicio. Stanners et al. (1 979) observaron que el TR de una palabra con prefijo (por ejemplo, retrieve) se ve facilitado por la presentación previa de trieve y de otra palabra que contenga el mismo prefijo (por ejemplo, remit). Este efecto de facilitación, no obstante, era menor que el obtenido cuando lo que se presentaba previamente era exactamente la misma palabra (por ejemplo, retrieve seguido por retrieve). La interpretación de estos resultados que hacen Stanners et al. es que las palabras con prefijo se almacenan tanto como palabras completas como en base a su raíz, existiendo interconexiones entre estas dos entradas.

Sin embargo, Taft ( 198 I) publica una respuesta a esto, recordando que el modelo que proponían él y Forster constaba de tres pasos: 1) quitar el prefijo re-, 2) localizar la entrada léxica trieve y 3) determinar que $r e$ - se puede combinar con trieve para formar una palabra. Sobre esta base, Taft (198I) argumenta que cuando ha de reconocerse como palabra el estímulo retrieve y se ha presentado precedido por trieve y remit, solamente se facilitan los dos primeros pasos del proceso de reconocimiento. Es decir, no se ha tomado la decisión de que re- puede combinarse con trieve y, por tanto, se dará un efecto de facilitación menor que cuando se han realizado los tres pasos (cuando previamente se presentaba la misma palabra).

De este modo, Taft ( $198 \mathrm{I}$ ) concluye que las palabras con prefijos no poseen una doble representación en el léxico, sino que están almacenadas una sola vez por medio de la raiz.

Por otro lado, Rubin et al. (1979) defendieron la idea de que las palabras con prefijo se reconocen a través de un proceso de descomposición morfológica solamente bajo ciertas circunstancias experimentales específicas. Estos autores partieron de la hipótesis coherente con el modelo de Taft y Forster- de que las palabras que, de hecho, no tienen prefijo, pero lo parece (palabras con pseudoprefijo) también serán sometidas a una segmentación morfológica consistente en la eliminación del prefijo. Por esta razón, el reconocimiento de una palabra con pseudoprefijo se verá demorado al descomponerla erróneamente en prefijo raíz. Rubien et al. demostraron que los TR empleados en responder a las palabras con 
pseudoprefijo eran, de hecho, más largos que los correspondientes a las palabras con prefijo. No obstante, este resultado se observa solamente cuando todas las no-palabras del experimento también tienen prefijos. Cuando las no-palabras carecen de prefijos, la diferencia entre las palabras con prefijo y con pseudoprefijo desaparece. A la vista de este resultado, los autores concluyen que la eliminación del prefijo no es un paso necesario del reconocimiento de este tipo de palabras, sino que es un fenómeno que sólo se observa cuando existen muchos ítems con prefijo entre los materiales del experimento. Por ello, afirman que los resultados de Taft y Forster son efectos de una estrategia, o que sólo se dan bajo determinadas circuntancias experimentales. En definitiva, lo que afirman es que cuando existen muchos estimulos con prefijo en el experimento, al sujeto le resulta rentable adoptar la estrategia de segmentación morfológica; mientras que, cuando éste no es el caso, el sujeto no necesitaria adoptar dicha estrategia. Lo cual está implicando el supuesto de que existe una doble representación de las palabras con prefijo (como argumentaban Stanners et al., 1979): el sujeto adoptará la estrategia de acceder a una u otra en función de las características de la situación experimental.

Para salir al paso de estas críticas, Taft ( $198 \mathrm{I}$ ) diseña tres experimentos, siendo de especial interés el segundo y el tercero. En el experimento 2, empleó una tarea de "naming" o denominación (véase Forster y Chambers, 1973). Se utilizaron solamente palabras (con prefijo y con pseudoprefijo). Dado que la mitad de las palabras tenian prefijo y la otra mitad no, no existe razón alguna para que los sujetos adoptaran una estrategia de descomposición morfológica frente a la de acceso a la palabra completa, si es que ambos procedimientos son posibles. Taft encontró que, incluso en este caso, las palabras con pseudoprefijos son erróneamente tratadas como si tuvieran un prefijo real.

En el experimento 3, no se utilizaron ítems con prefijo. I.a hipótesis era la siguiente: si las palabras con pseudoprefijo son erróneamente tratadas como si tuvieran prefijo y esto lentifica el acceso léxico de tales palabras, es de esperar que den lugar a tiempos de reacción mayores que las palabras sin prefijo, a las que puede accederse como unidades inmediatamente. Utilizando una tarea de «naming», se confirmó esta hipótesis. A partir de los resultados, se ve que a las palabras con pseudoprefijo no se accede inmediatamente tratándolas como unidades, sino que, efectivamente, son incorrectamente tratadas como si lo tuvieran, incluso cuando en el experimento no existen palabras con prefijo.

Del Viso (1984) llevó a cabo varios experimentos en castellano con el propósito de determinar el «status» léxico de palabras relacionadas morfológicamente y comprobar si los resultados coincidían con los obtenidos en otros idiomas. En un primer experimento, se utilizaron nombres con inflexiones (concretamente de número, manteniendo constante el género). Con una tarea de decisión léxica (véase, por ejemplo, García-Albea, 1982, págs. 207-208), se encontraron TRs menores en las respuestas correspondientes a los nombres cuando se presentaban en singular ( $\sin$ que este efecto pudiera 
explicarse solamente en virtud del número de letras - menor en singular - ni de la frecuencia de uso - que suele ser mayor para el singular-, ya que las palabras utilizadas fueron igualadas en longitud y frecuencia). De una forma tentativa, puede decirse que los resultados indican que la forma base de los nombres está mejor representada por el singular y que, en el proceso de acceso al léxico, cuando las palabras se presentan en plural es posible que el sujeto efectúe algún tipo de descomposición morfológica para dar con la entrada léxica correspondiente. Además, nuestros resultados muestran que, tomando la frecuencia de uso como un buen predictor del tiempo que se invierte en reconocer una palabra (para una revisión de este tema, véase García-Albea, 1982 ; págs. 208-209 y Garcia-Albea y cols., 1982, paǵs. 32-36), la frecuencia del singular es tan buen predictor como la del plural cuando la palabra se presenta en plural, no observándose, además, el efecto complementario.

En los experimentos siguientes, en lugar de inflexiones, se utilizaron palabras derivadas, dadas las diferencias lingüísticas (véase, por ejemplo, Bosque, 1982) y psicológicas (por ejemplo, Murrell y Morton, 1974; Mac Kay, I978) existentes entre flexión y derivación. De hecho, Chomsky (1970) habia sugerido ya que inflexiones y derivaciones podrían presentar diferencias en cuanto al modo de almacenamiento en la memoria.

Utilizando también tareas de decisión léxica y basándose en el fenómeno de «priming», o facilitación por presentación previa (para una revisión del tema, véase Del Viso, I984, págs. I Io- I I3), la lógica de estos experimentos era la siguiente: suponiendo que el reconocimiento de una palabra se ve facilitado (es decir, el TR es menor) por la presentación previa de esa misma palabra, se trataba de comprobar si también se producía este efecto, y en qué medida, cuando lo que se presentaba previamente no era exactamente la misma palabra, sino otra relacionada morfológicamente.

En los dos primeros experimentos de esta serie, los estímulos se presentaron de forma visual; en el tercero, auditivamente. En el primero de ellos, se comprobó el efecto facilitador que produce tanto la presentación previa de la misma palabra como la presentación previa de una forma derivada de la misma, aunque los efectos en uno y otro caso no eran equivalentes, siendo superior el primero al segundo. La interpretación de este resultado, así como la del anterior, parece apoyar el modelo propuesto por Taft y Forster (1975); es decir, la hipótesis de la descomposición morfológica y, por tanto, apunta hacia un modelo de reconocimiento centrado en la forma base de las palabras.

El siguiente experimento puede considerarse como complementario del que acabamos de exponer. En este caso, se trataba de comprobar qué sucedía cuando lo que se presentaba en primer lugar era la foma base (no derivada) procediendo a una palabra derivada de ella. También aquí se encontraron los efectos de facilitación esperados, pero lo que interesaba especificamente era comprobar los efectos de facilitación encontrados en el experimento anterior y en éste; es decir, la facilitación que una palabra derivada ejerce sobre el posterior reconocimiento de su forma base correspondiente y la 
facilitación que produce la presentación previa de la palabra base sobre el reconocimiento de sus formas derivadas. Los resultados no arrojaron diferencias significativas entre ambos efectos, lo cual parece indicar que la relación entre ambos tipos de formas verbales (base y derivada) sería simétrica, no pudiendo hablarse de una dependencia unilateral a efectos de reconocimiento.

Los resultados parecen más bien contrarios a la hipótesis de la existencia de entradas léxicas separadas para las palabras relacionadas morfológicamente. Y, desde luego, son compatibles con la hipótesis de la descomposición morfológica. Parece claro que si la presentación previa de una palabra (sea base o derivada) facilita el reconocimiento de otra relacionada morfológicamente, las entradas léxicas de ambas no podrían ser independientes, sino que tendrían que estar interconectadas o relacionadas de algún modo.

Por último, se compararon en un mismo experimento los dos efectos de facilitación ya comentados, tratándose además de poner de manifiesto si los resultados obtenidos con presentación visual eran generalizables a la modalidad auditiva de reconocimiento. Los resultados indicaron, en primer lugar, que efectivamente se da un efecto de facilitación en el reconocimiento cuando previamente se ha presentado bien la misma palabra o bien una relacionada morfológicamente. En segundo lugar, que el efecto de facilitación es mayor cuando lo que se presenta en primer lugar es exactamente la misma palabra que la que vendrá posteriormente. $Y$ en tercer lugar, que no existen diferencias entre la facilitación que produce la palabra derivada sobre la base y la que produce ésta sobre aquélla. Esto viene a confirmar, en la modalidad auditiva, los resultados que ya se habían obtenido en la modalidad visual en los dos experimentos anteriores.

\section{DATOS EXPERIMENTALES EN EL AREA DE LA PRODUCCION}

En el área de la producción, es de destacar la labor de D. Mac Kay, a partir de 1974. En la investigación de 1976, Mac Kay estudia los procesos de recuperación léxica de verbos irregulares en pasado (por ejemplo, teach-taught) a la luz de las dos perspectivas generales sobre la organización del léxico interno: lo que él denomina hipótesis derivacional e hipótesis de independencia. Aunque con una denominación propia de este autor, ambas hipótesis vienen a corresponderse con las dos que venimos mencionando. Recapitulando en pocas palabras, la hipótesis derivacional propone que raíces y sufijos poseen representaciones independientes en el léxico, de tal modo que formas como taufht se generan aplicando una serie de reglas a la forma base teach; la hipótesis de independencia, por otro lado; postula que todas y cada una de las palabras poseen representaciones separadas en la memoria léxica.

Aunque expone algunos argumentos $\mathrm{y}$ datos experimentales a favor de la hipótesis derivacional (véase Mac Kay, 1976, páginas $169-170$ ), el autor reconoce la existencia de ciertas razones a 
priori que apoyan la hipótesis de independencia en el caso de los pasados irregulares, dado el cambio fonético y ortográfico que éstos implican en la mayoría de los casos. El argumento central se basa en que, dados que los pretéritos irregulares son pocos, pero de uso muy frecuente, resultaria ciertamente económico aprenderlos y almacenarlos como unidades independientes, en lugar de adquirir una serie de reglas, numerosas y complejas, para formar los pasados irregulares a partir de sus formas base correspondientes.

Por estas razones, Mac Kay ( 1976 ) diseñó dos experimentos para adoptar una u otra hipótesis. ..En ambos, a los sujetos se les presentaba auditivamente un verbo base y su tarea consistia en producir el pretérito correspondiente en el menor tiempo posible. El tiempo de producción era la variable dependiente, mientras que la independiente era los cuatro tipos de estímulos utilizados: $\mathrm{I}$. Verbos regulares en -t (por ejemplo, walk-walked: $/ \mathrm{w} \asymp \mathrm{k} /-/ \mathrm{w} \times \mathrm{kt} /$ ). 2 . Verbos irregulares en -t (por ejemplo, lose-lost: /luxz/ - /lost/). 3. Cambio de vocales simple (por ejemplo, dig-dug: /dig/ - $/ \mathrm{d} \wedge \mathrm{g} /$ ). 4. Cambio de vocales complejo (por ejemplo, bide-hid:/haid/ - /hid/). Según la hipótesis derivacional, cabría esperar tiempos de producción más breves para los verbos tipo I que para los verbos tipo 2, y más cortos también para los del tipo 3 que para los del tipo 4 . La hipótesis de independencia, en cambio, postularia que no existen diferencias entre estos materiales, dado que los pasados se recuperan directamente del léxico sin necesidad de aplicar reglas de cambio vocálico ni de ningún otro tipo. En general, los datos - tanto los correspondientes al tiempo de producción como al tipo de errores cometidos - apoyan la hipótesis derivacional; aunque, hablando en sentido estricto, los resultados solamente indican que los hablantes pueden emplear reglas derivativas para formar los pretéritos. Queda abierta la cuestión de si habitualmente emplean este tipo de reglas en la produción de oraciones; si bien, existen datos basados en el estudio de los errores espontáneos en el habla (Fromkin, 1973, 1980; Mac Kay, 1974) que indican que estas reglas desempeñan, de hecho, un papel importante en el habla continuada. También queda aún abierta la cuestión de qué forma adoptan estas reglas y cómo se aprenden.

En otro trabajo, Mac Kay (1978) estudia la producción de nombres derivados de verbos, en la misma linea de sus investigaciones anteriores. La tarea era la misma que en los experimentos de 1976, manipulándose, asimismo, la complejidad de las reglas derivativas que habían de aplicarse en cada caso. Los tiempos de producción y los errores se distribuyeron en función de la complejidad derivativa, lo cual apoya de nuevo la hipótesis derivacional para el caso de palabras derivadas (recordemos que, en los experimentos de 1976, las palabras que los sujetos tenían que producir eran inflexiones de los verbos base que se les presentaban) que cambian la categoría gramatical de las palabras a partir de las que son producidas (en este caso, de verbo a nombre).

Finalmente, Mac Kay (1979) estudia los procesos de producción de palabras y oraciones, distinguiendo - en el caso de las palabrasafijos derivativos e inflexivos. Los análisis de un corpus de errores 
en alemán y en inglés apoyan la tesis de que las palabras complejas se generan a partir de sus formas base correspondientes mediante la aplicación de reglas, e indican que las raices de las palabras, los prefijos y los sufijos se almacenan por separado en el léxico, junto con un «marcador» que define su función sintáctica en la combinación con otros componentes de la palabra. Mac Kay elabora, finalmente, un modelo de los procesos léxicos en la producción del lenguaje que consta de tres etapas: una primera de inserción léxica, donde las formas léxicas abstractas se introducen en la oración basándose en características sintácticas y semánticas abstractas; a continuación, tiene lugar una etapa de aplicación de reglas de concordancia y flexivas, derivativas y fonológicas que darán lugar a la cadena fonética; por último, se da una etapa de "output serial», en virtud de la cual la cadena fonética se transforma en órdenes motoras (articulatorias) ordenadas serialmente. (Para una revisión más extensa de este modelo, véase Mac Kay, 1979, págs. 492-495.)

Si bien, Mac Kay ha sido el autor que más ha trabajado sobre el tema de la representación léxica de las palabras relacionadas morfológicamente en el área de la producción, existe al menos otro trabajo en torno a esta cuestión. Van der Molen y Morton (1979) estudiaron la representación de memoria de los nombres con inflexiones. Utilizaron una tarea de recuerdo de listas, constituidas cada una de ellas por seis nombres, de los cuales uno o dos aparecían en plural. Los autores encontraton un número considerable de errores consistentes en que, en el recuerdo escrito de los sujetos, desaparecía el morfema del plural (es decir, se recordaban en singular nombres que habían sido presentados en plural). Se llegó a la conclusión de que el código para realizar esta tarea estaba basado en la estructura morfológica, no en la palabra como un todo.

\section{DATOS EXPERIMENTALES EN EL AREA DE LA ADQUISICION}

Algunos estudios sobre adquisición del lenguaje también aportan datos sobre el tema que nos ocupa. Aunque escasos, existen varios trabajos cuyo objetivo es estudiar el proceso por el que se aprenden las reglas morfológicas.

Parece que la capacidad para descomponer o analizar morfológicamente palabras complejas es uno de los aspectos del lenguaje que más tarde se adquieren. En un estudio ya clásico, Berko (1958) demostró que niños pequeños (de cuatro a siete años) dominaban ya las reglas inflexivas (los plurales y las formas regulares del pasado de los verbos), pero no las derivativas ni las reglas morfológicas de las palabras compuestas.

Más recientemente, Derwing y Baker (1977, 1979) emplearon tareas de juicio para estudiar la adquisición de la morfología. La muestra de sujetos estaba constituida por 40 niños (de ocho a doce años), 28 adolescentes (de trece a diecisiete) y 27 adultos, en el estudio de 1977 , y solamente por niños en el de 1979. Lo que hacian era preguntar a los sujetos si ciertas plabras (por ejemplo, teach-tea- 
cher, dirt-dirty) estaban relacionadas entre si, si una procedía de la otra, así como que evaluaran el grado de seguridad en sus respuestas. Observaron que existe una capacidad para reconocer o identificar morfemas que va aumentando con la edad. También encontraron que los niños más pequeños presentan una tendencia a basarse en consideraciones fonéticas para emitir sus juicios.

Finalmente, quisiéramos mencionar un último estudio que toca de lleno el tema del aprendizaje de las reglas morfológicas. Nos referimos al trabajo de-Freyd y Baron (1982). Estos autores plantean dos hipótesis explicativas de la adquisición de las palabras morfológicamente complejas, que se corresponden básicamente con las dos que venimos manejando acerca de la representación léxica de este tipo de palabras. La primera de ellas propone que tales palabras se aprenden como unidades completas, es decir, sin analizar. La segunda, en cambio, postula que se adquieren a través de la aplicación de las reglas de formación de palabras. Los autores toman partido por esta segunda hipótesis, argumentando que las consecuencias que se siguen de la misma pueden resultarle muy útiles al niño que está aprendiendo una lengua. En este sentido, proponen que, al aprender una determinada regla de derivación, no es que cambie el modo de almacenamiento de las palabras, sino que quizá lo que ocurre es que se incorpora un nuevo nivel de organización que permite realizar análisis morfológicos, aparte de otros análisiss que pueden hacerse de las palabras. Este análisis puede resultar muy útil para ciertas tareas lingüísticas -aunque no para todas-; por ejemplo, puede facilitar el acceso a ciertas palabras relacionadas o el aprendizaje de palabras nuevas.

Lo que Freyd y Baron hicieron fue leer palabras - la mayoria derivadas - en voz alta a los niños y pedirles que escribieran una definición. Aparte de ciertas diferencias individuales en cuanto a los mecanismos empleados para aprender palabras derivadas - muy importantes, pero no demasiado relevantes para nuestros propósitos-, observaron que, en general, los niños presentaban una dificultad mayor en relación con las palabras complejas. Los autores atribuyen este hecho a la tendencia a analizar morfológicamente este tipo de palabras, junto con un relativo desconocimiento de los efectos de los afijos sobre el significado de una palabra. Por otra parte, sus resultados vienen a confirmar la hipótesis de que las palabras morfológicamente complejas están representadas en el léxico como subentradas de la forma base, en cuya representación se incluyen también las reglas necesarias para generar las posibles variaciones.

\section{DATOS EN EL AREA DE LA PATOLOGIA}

Durante largos años, no se han tenido en cuenta los datos de patologías orgánicas en las teorías sobre el funcionamiento normal del lenguaje. Este hecho refleja, al menos en parte, un escepticismo en relación con la relevancia de la afasiologia para la psicolingüística, dado que el punto de vista más extendido era que la función del 
lenguaje en el individuo con una lesión cerebral era producto de un sistema "aberrante» que no reflejaba el estado normal.

Sin embargo, el estudio de la áfasia para atender el procesamiento normal del lenguaje ha recibido un creciente interés a lo largo de los últimos años (Goodglass y Blumstein, 1973; Fodor, Bever y Garrett, 1974; Zurif y Caramazza, 1976; Kean, 1977, 1980; Bradley, 1978; Caramazza y Berndt, 1978; Bradley, Garret y Zurif, 1980; Sánchez Bernardos, $1982,19^{83}$ ). Este interés surgió a partir de la observación de que las lesiones en distintas áreas del hemisferio izquierdo son bastante selectivas en sus efectos, existiendo, además, una gran consistencia en cuanto a cómo afectan al lenguaje las distintas lesiones (Bradley, Garret y Zurif, 1980).

En definitiva, y por decirlo en pocas palabras, los psicolingüistas volvieron sus ojos hacia la afasiología pensando que si es cierto que el lenguaje patológico refleja de algún modo las capacidades que existían antes de la lesión, se podrían hacer inferencias acerca de la organización de los procesos normales del lenguaje a partir de las funciones deterioradas en comparación con aquellas que aún quedan preservadas.

Concretamente en relación con nuestro trabajo, el hecho más destacable encontrado en los estudios sobre afasia es que la morfología inflexiva está casi completamente ausente en el habla agramática (dificultad con las palabras funcionales), mientras que se encuentra preservada en la anomia (dificultad con las palabras de contenido). Está preservada incluso en los casos de afasia de Wernicke que presentan jerga, donde la estructura fonológica de los elementos léxicos está gravemente afectada.

Este aspecto ha sido estudiado con detenimiento en un reciente trabajo de Saffran, Schwartz y Marin (1980), en el ámbito de la producción. Los rasgos caracteristicos del agramatiso propio de los pacientes con afasia de Broca pueden agruparse en los siguientes:

I. Una ausencia de morfemas gramaticales, tanto libres como ligados, que da al habla agramática su carácter de «telegráfica». La omisión de las palabras funcionales y de los afijos inflexivos no sólo se manifiesta en el habla espontánea, sino también en tareas de completar oraciones (Goodglass et al., 1972; Gleason et al., 1975) e incluso en tareas de mera repetición (Goodglass, 1968).

2 Una simplificación - $\mathrm{y}$ en los casos más graves, ausencia total- de la estructura sintáctica de la oración. Este déficit sintáctico puede, a veces, quedar paliado por la utilización de estrategias semánticas en la construcción de las oraciones. Por ejemplo, Saffran et al. (1976) observaron que la actuación de los afásicos agramáticos en tareas de producción y ordenamiento de oraciones mejoraba notablemente cuando los objetos diferían en la categoria animadoinanimado (su ejecución era mejor en la oración "The girl runs to the bouse» que en "The key is in the suitcase»). Este resultado indica que los pacientes agramáticos se apoyan en factores pragmáticos para ordenar o producir una oración y no en mecanismos basados en reglas sintácticas. Se observa que el objeto de mayor relevancia 
semántica, casi siempre un objeto animado, aparece en primer lugar, desempeñando la función de sujeto de la oración.

3. Un sesgo léxico hacia las palabras de contenido, en especial hacia los nombres concretos y que se corresponden con una imagen gráfica. Los verbos, cuando se dan, tienden a ser no marcados ni para la persona (por ejemplo, "The girl study", cuando deberia ser "The girl studies») ni para el tiempo (por ejemplo, "The boy is climb the fence», cuando debería ser "The boy is climbing the fence»). A este respecto, también existen datos sobre pacientes agramáticos alemanes, que tienden a emplear el infinitivo en lugar de las formas flexivas apropiadas (Goodglass y Geschwind, 1976).

4. Además de las caracteristicas morfológicas y estructurales descritas, estos pacientes presentan una dificultad articulatoria que se traduce en un lenguaje producido con esfuerzo, pobremente articulado y carente de entonación. Es interesante destacar, en inglés, la tendencia a sustituir las consonantes no marcadas (por ejemplo, finales de palabra que no se pronuncian, como $/ \mathrm{p} / \mathrm{y} / \mathrm{t} /$ ) por consonantes marcadas (finales sonoros, como $/ \mathrm{b} / \mathrm{y} / \mathrm{d} /$ ).

Por otra parte, algunas investigaciones realizadas en el terreno de la comprensión han observado que los afásicos agramáticos presentan déficit similares en este área (véase el trabajo de Zurif y Caramazza, 1976).

Existen distintas explicaciones alternativas para dar cuenta de todos los déficits observados en el agramatismo, pero, por razones de espacio, no vamos a detenernos sobre ellas (puede verse la discusión de Saffran et al., 1980, págs. 233-237). Sí, en cambio, quisiéramos señalar que, tomando todas estas observaciones en conjunto, se encuentra una especial dificultad del paciente agramático en la producción de los afijos flexivos - no se dispone, por el momento, de datos acerca de los derivativos-. La primera conclusión que puede extraerse de estas observaciones, sin riesgo de caer en falsas interpretaciones, es que las variables morfológicas desempeñan una función específica en la producción del lenguaje (posiblemente también en la comprensión, aunque existen menos datos) y que pueden verse seriamente afectadas en determinados casos de lesión cerebral.

\section{CONCLUSIONES}

Los resultados obtenidos en los distintos trabajos que hemos revisado ponen de manifiesto la relevancia del papel de las variables morfológicas de cara a investigar la organización del léxico interno y el acceso al mismo.

En este campo de estudio, vienen manejándose dos grandes hipótesis, contrapuestas entre sí, para describir la representación léxica de las palabras relacionadas morfológicamente. Una de ellas (hipótesis de independencia, según la terminología de Mac Kay, 1976), propone que cada palabra (primitiva o derivada, con sufijos marcados o no) posee su propia entrada léxica independiente de las 
entradas de otras palabras relacionadas morfológicamente. La segunda (hipótesis derivacional, también siguiendo a Mac Kay) postula la existencia de una sola entrada léxica común a todas las palabras pertenecientes a una misma familia.

Si bien la hipótesis de independencia ha recibido algún apoyo experimental, parece que los argumentos que proponen sus partidarios para explicar los resultados son rebatibles, y que estos últimos pueden interpretarse en un sentido alternativo. Por su parte, la hipótesis de la entrada léxica común parece sustentarse en numerosos resultados experimentales, no sólo en inglés, sino también en otros idiomas, entre ellos, el castellano. Es de destacar, en otro orden de cosas, la convergencia obtenida entre los resultados de los experimentos con presentación visual y auditiva, lo cual constituye un apoyo a la idea de que las propiedades fundamentales de ambos modos de procesar el lenguaje serían muy similares a nivel central, como ya se había sugerido (Garrett, 1978).

A modo ya de conclusiones generales, podemos decir que las variables morfológicas no obedecen solamente a distinciones que efectúe el lingüista en su tarea, sino que la estructura morfológica desempeña un papel importante en la organización de las palabras en el sistema cognitivo del hablante-oyente. Abundando en esta idea, tanto los resultados obtenidos con palabras que presentan inflexiones como con palabras derivadas ponen de manifiesto que el reconocimiento de palabras relacionadas morfológicamente obedece a principios computacionales que tienen en cuenta dicha relación.

De cara a la forma de organización del léxico interno, la representación de las palabras parece regirse por un principio de economía de recursos que permite reconocer las palabras relacionadas morfológicamente a través de una entrada léxica común - caracterizada por un alto grado de abstracción con respecto a las formas superficiales de las mismas - que contiene información suficiente acerca de todas las posibles realizaciones concretas de una palabra. La entrada léxica se activaría frente a cada ocurrencia de estas últimas. Se puede pensar que en el análisis preliminar del «input» verbal, se ha de llevar a cabo una segmentación de tipo morfológico para utilizar la raíz de la palabra en el acceso al léxico.

Para terminar, no quisiéramos dejar de señalar que, a pesar de la abundancia relativa de datos, como suele suceder, aún no están suficientemente claras todas las cuestiones posibles. A modo de ejemplo, y por apuntar algunas vias de investigación futuras, seria interesante estudiar comparativamente los procesos implicados con respecto a inflexiones y derivaciones, al menos en castellano, puesto que en algunos trabajos en inglés (por ejemplo, Murrell y Morton, 1974; Stanners, Neiser, Hernon y Hall, 1979) se han encontrado diferencias en el comportamiento de palabras con estos dos tipos de sufijos. Por otra parte, también sería conveniente diseñar experimentos "cruciales" que nos permitieran decidir, más o menos definitivamente, por un modelo de representación léxica en contra de otros. 


\section{Referencias}

BERKO, J. (1958): «The child's learning of English morphology". Word, 14, 1 50-177.

BOSQUE, I. (1982): «La morfología». En F. ABAD y A. GARcía BARRIO (Eds.): Introducción a la Lingüistica. Madrid, Alhambra.

BRADLEY, D. C. (1978): Computational distinctions of vocabulary type. Tesis doctoral no publicada. M. I. T.

BraDley, D. C. (I979): “Lexical representation of derivational relation». En M. ARONOFF y M. L. KEAN (eds.): Juncture. Cambridge, Mass.: M. I. T. Press.

Bradi.eY, D. C.; GarretT,'M. F., y ZURIF, E. (i980): "Syntactic deficit in Broca's aphasia». En D. CaPl.an (ed.): Biological Studies of Mental Processes. Cambridge, Mass., M. I. T. Press.

Caramazza, A., y Berndt, R. S. (1978): «Semantic and syntactic processes in aphasia: A review of the literature». Psychological Bulletin, 8, 898-918.

ChOMSKy, N. (1970): «Remarks on nominalizations». En R. JaCOBS y P. Rosenbaum (eds.): Readings in English Transformational Grammar. Waltham, Mass., Ginn.

DERWING, B. L., y BAKER W. J. (I 977): "The psychological basis for morphological rules". En J. Macnamari (ed.): Language and thought. New York, Academic Press.

DERWIN, B. L., y BAKER, W. J. (1979): «Recent research on the acquisition of English morphology». En P. Fletcher y M. GARMAN (eds.): Language acquisition: Studies in first langwage development. Cambridge, Cambridge University Press.

FODOR, J. A.; Beber, T. G., y GARRETT, M. F. (1974): The psychology of language. Nueva York: McGraw Hill.

FORSTER, K. I. (1976): "Accessing the mental lexican". En E. W. WALKER y R. J. WALES (Eds.): New approaches to language mechanisms. Amsterdam, North Holland.

FORSTER, K. I., y CHAmbers, S. M. (1973): "Lexical access and naming time». Journal of Verbal Learning and Verbal Bebavior, $12,627-635$.

FREYD, P., y BARON, J. (1982): «Individual differences in acquisition of derivational morphology». Journal of Verbal Learning and Verbal Behavior, 21, 282-299.

FromkIN, V. A. (1973): Speecb errors as linguistic evidence. Nueva York, Mouton.

FROMKIN, V. A. (1980): "Introduction». En V. A. FROMKIN (ed.): Errors in linguistic performance. Nueva York, Academic Press.

GARCIA-AI.BEA, J. E. (1980): "Variables estructurales en el reconocimiento visual de palabras». Informes del Departamento de Psicologia General. (Univ. Complutense de Madrid), 3/6, 3-22.

García-AibeA, J. E. (1982): "Algunos aspectos en el estudio del procesamiento del lenguaje». En I. Deicladux y J. Seone (eds.): Psicologia cognitiva y procesamiento de la información. Madrid, Pirámide.

GARCíA-AI.BEA, J. E. (1984): Syllabic structure and stress in the segmentation of the visual input for word recognition in Spanish. Trabajo presentado en la European Psycholinguistic Association Workshop «Cross-Linguistic Studies of Morpho-phonological Processing». París, junio 1984.

Garcia-Ai.bea, J. E; SÁnchez-Casas, R. M., y Dei. Viso, S. (1982): «Efectos de la frecuencia de uso en el reconocimiento de palabras». Investigaciones Psicológicas, $I, 24-63$.

GarretT, M. F. (1978): «Word and sentence perception». En R. HEID, H. W. Liebowitz y H. L. TEuber (eds.), Handbook of sensory Pbysiology. Vol., VIII: Perception. Berlin, Springer-Verlag.

GibSON, E. J., y GUINET, L. (1971): "Perception of inflections in brief visual presentations of words». Journal of Verbal Learning and Verbal Bebavior, 10, 182-189.

Gi.eason, J. B.; GoOdglass, H.; Green, E.; ACKerman, N., y Hyde, M. (1975): "The retrieval of syntax in Broca's aphasiay. Brain and Language, 12, $451-47 \mathrm{I}$.

GoODGI.Ass, H. (1968): "Studies on the grammar of aphasics». En S. ROSENBERG, y J. KOPI.IN (eds.), Developments in applied psycholinguistic research. New York, Mac Millan.

GoOdgi.ASS, H., y BI.UMSTEIN, S. (1973): Psycholinguistics and apbasia. Baltimore, John Hopkins University Press.

Goodglass, H, y Geschwind, N. (1976): "Language disorders (aphasia)». En E. C. CarteretTE y M. Friedman (eds.), Handbook of perception VII. New York, Academic Press.

GoOdglass, H.; GieASON, J: B.; BERNHOlTZ, N. A., y HYDE, M. (1972): «Some linguistic structures in the speech of a Broca's aphasia». Cortex, 8, 191-212.

HOIYOAK, K. J.; GI.ASS, A. L., y MAH, W. A. (1976): «Morphological structure and semantic retrieval». Journal of Verbal Learning and Verbal Bebavior, 15, $235-247$.

JARVELLA, R. J., y SNODGRASS, J. G. (1974): «Seeing ring in rang and retain in retention: On recognizing stem morphemes in printed words". Journal of Verbal Learning and Verbal Bebavior, 13, 590-598.

KEAN, M. L. (1977): «The linguistic interpretation of aphasic syndromes: Agrammatism in Broca's aphasia, an example». Cognition, $5,9-46$.

KEAN, M. L. (1980): "Grammatical representations and the description of language processes». En D. CAPl.AN (ed.): Biological studies of mental processes. Cambridge, Mass., M. I. T. Press.

KINTSCH, W. (1972): "Abstract nouns: Imagery versus lexical complexity». Journal of Verbal Learning and Verbal Bebavior, II, 55-61.

Lukatela, G.; Mandio, Z.; Gi.igorijević, B.; Kostić, A.; Savić, M., y Turvey, M. T. (1978): Lexical decision for inflected zouns. Haskins Laboratories: Status Report on Speecb Researcb SR-J3, vol. 1, 65-72. 
M^C K^Y, D. G. (1974): Derivational rules: Dead souls or living people in the perception, production and storage of words? Trabajo presentado en el Psycholinguistic Circle of New York. Abril.

MAC KAY, D. G. (1976): “On the retrieval and lexical structure of verbs». Journal of Verbal Leerning and Verbal Bebavior, 15 , $169-182$.

MAC KАY, D. G. (1978): «Derivational rules and the internal lexicon». Journal of Berbal Learning and Verbal Bebavior, 17, 61-71.

MAC KAY, D. G. (1979): “Lexical insertion, inflection and derivation: creative processes in word production". Journal of Psycbolinguistic Research, 8, 477-498.

Manel.iS, L., y TharP, D. (1977): "The processing of affixed words". Memory and Cognition, 5, 690-695.

MASSAR(), D. W. (1974): «Perceptual units in speech recognition». Journal of Experimental Psychology, 102, $199 \cdot 208$.

Massaro, D. W.; TAylor, G. A.; Venezky, R. L.; Jastrzembski, J. E., y Lucas, P. A. (1980): Letter and word perception: The role of ortbographic structure and visual processing in reading. Amsterdam, North Holland.

Mehi.er, J.; Dommergues, J. Y.; Frauenfei.der, U., y SEgul, J. (1981): "The syllable's role in speech segmentation». Journal of Verbal Learning and Verbal Bebavior, 20, 298-305.

MURRei, G. A., y MORTON, J. (1974): "Word recognition and morphemic structure». Journal of Experimental Psychology, 102, 963-968.

OI.DFIELD, R. C. (1966): "Things, words and the brain". Quarterly Journal of Experimental Psychology, I8, 340-353.

RubiN, G. S.; BeCKer, C. A., y Freeman, R. H. (1979): «Morphological structure and its effect on visual word recognitions. Journal of Verbal Learning and Verbal Bebavior, 18, 757-767.

SAFFr AN, E. M.; SchwARTZ, M. F., y MARIN, O. S. M. (1976): «Semantic mechanisms in paralexia». Brain and Language, 3, 255-265.

SÁFFR AN, E. M.; SCHWARTz, M. F., y MARIN, O. S. M. (1980): «Evidence from aphasia: Isolating the components of a production models. En B. BUTTERWORTH (ed.): Language production. Vol. I. Nueva York, Academic Press.

SÁNCHEZ BERNARDOS, M. L. (1982): Una nueva aproximación al estudio de la afasia: la alteración de los niveles de procesamiento del lenguaje. Comunicación presentada en el VII Congreso Nacional de Psicología. Santiago de Compostela.

SÁnCliez Bernardos, M. L. (1983): Elaboración de una Escala Española para la Evaluación de la Afasia. Memoria de licenciatura no publicada. Universidad Complutense de Madrid.

SÁNCHEz-CASAS, R. M. (1982): El papel de la categoría gramatical en el acceso al léxico interno. Memoria de licenciatura no publicada. Universidad Complutense de Madrid.

SMITH, P. T., y STERIING, C. M. (I 982): «Factors affecting the perceived morphemic structure of written words». Journal of Verbal Learning and Verbal Behavior, 2I, 704-721.

SNOdgrass, J. G., y JaRVel..A, R. J. (1972): «Some linguistic determinants of word classification times». Psychonomic Science, 27, 220-222.

SpoEHR, K. T., y Smith, E. E. (1973): "The role of syllables in perceptual processing». Cognitive Psychology, S, 71-89.

StanNers, R. F.; Neiser, J. J; Hernon, W. P., y HAI.l, R. (1979): «Memory representation for morphologically related words". Journal of Verbal Learning and Verbal Bebavior, 18, 399-4I 2.

StANNERS, R. F.; NeISER, J. J., y PAINTON, S. (I979): «Memory representation for prefixed words». Journal of Verbal Learning and Verbal Bebavior, 18, 733-743.

TAFT, M. (1979): "Lexical access via an orthographic code: The Basic Orthographic Syllabic Structure (BOSS)m. Journal of Verbal Learning and Verbal Bebavior, 18, $2 \mathrm{I}-39$ (a).

TAFT, M. (1979): «Recognition of affixed words and the word frequency effect». Memory and Cognition, 7 , $263-272$ (b).

TAFT, M. (198I): «Prefix stripping revisited». Journal of Verbal Learning and Verbal Bebavior, 20, 28 9-297.

TAFT, M., y FORSTER, K. I. (1975): «Lexical storage and retrieval of prefixed words». Journal of Verbal Learning and Verbal Bebavior, 14, 638-647.

TAFT, M., y FORSTER, K. I. (I976): «Lexical storage and retrieval of polymorphemic and polysyllabic words". Journal of Verbal Learning and Verbal Bebavior, 1s, 607-620.

VAN DER MOIEN, H., y MORTON, J. (1979): «Remembering plurals: Unit of coding and form of coding during serial recalls. Cognition, 7, 35-47.

DEI. VISO, S. (1984): El papel de las variables morfológicas en la organización del léxico interno. Memoria de licenciatura no publicada. Universidad Complutense de Madrid.

WhEElER, D. D. (1970): «Processes in word recognition». Cognitive Psychology, 1, 59-8 s.

Zurif, E., y CaramazzA, A. (1976): «Psycholinguistic structures in aphasia: Studies in syntax and semantics». En H. WhITAKER y H. A. WhITAKER (eds.): Studies in Neurolinguistics. Nueva York: Academic Press.

LeveI.T, W. J. M. (1978): «A Survey of Studies in Sentence Perception», 1970-1976. En W. J. M. LEVEI.T y G. B. FIORES D'ARCAIS (1964): Studies in the Perception of Language. J. Wiley and Sons, $197^{8 .}$

Poll.ACK, I., y PiCKeET, J. M. (1964): "Intelligibility of excerpts from fluent speech: Auditory versus structural context". Journal of Verbal Learning and Verbal Bebavior, 3, 79-84.

SACHS, J. (1967): "Recognition memory for syntactic and semantic aspects of connected discourse", en Perception and Psychophysics, 2, 437-442. 
SeArLe, J. (1969): Speech acts: An Essay in the Pbilosopby of Language. Cambridge University Press. SELESKOVITCH, D. (1968): L'Interprete dans les conférences internationales. París, Minard.

STEINER, G. (1979): «Comments on Louis Kelly's book The True Interpreter». The London Review of Books, 22 de noviembre de 1979 .

Steiner, G. (1975): After Babel, Aspects of Language and Translations. Oxford University Press.

Certeau, Michel De (1981): "La lecture absolue», en Problemes actuels de la lecture. Clancier-Guenaud. Paris, 66.

\section{Resumen}

En este articulo se revisan los principales estudios acerca del papel que pueden desempeñar las variables de tipo morfológico en la organización del léxico interno y en el acceso a la información contenida en el mismo. Más en concreto, se trata de ver basta qué punto las relaciones derivativas entre palabras contribuyen a una simplificación del inventario mental de entradas léxicas. Para ello, se examinan las dos bipótesis alternativas fundamentales que ban sido propuestas a este respecto: la bipótesis de independencia, según la cual a cada palabra le corresponderia una entrada léxica distinta; y la hipótesis derivacional, según la cual babría una misma entrada léxica para cada familia de palabras relacionadas morfológicamente. Los datos procedentes de las distintas áreas de la investigación psicolingüistica (comprensión, producción, adquisición y trastornos del lenguaje) parecen apoyar esta segunda bipótesis, aunque se hace notar la falta de resultados cruciales que permitan descartar la bipótesis de independencia o alguna versión modificada de la misma. Sin embargo, aun en el caso de quie bubiera entradas léxicas distintas, seria preciso postular algún mecanismo adicional que diera cuenta de la información morfológica que utiliza el sujeto en el procesamiento de palabras.

\section{Summary}

This paper reviews a series of studies about the role played by morphological factors in the organization of the internal lexicon and in the access to the information contained in it. In particular, the question faced bere is to what extent the derivational relations among words may contribute to a simplification of the mental inventory of lexical representations. For this purpuse, the two main alternative bypothesis which babe been proposed are considered: the bypothesis of independence, where each particular word bas its own lexical entry; and the derivational bypothesis, where the same lexical entry would represent a whole family of morphologically related words. Data coming from different areas of psychplinguistic research (comprebension, production, acquisition and language disorders) seem to support the second of these bypothesis, although there are no crucial results which allow to discard the bypotbesis of independence or some modified version of it. However, even in the case of separated lexical entries, some other mechanism ought to be postulated in order to account for the morphological information use in word processing. 